\title{
Magellanic Diffuse Interstellar Bands and Carbon Chemistry
}

\author{
P. Ehrenfreund ${ }^{1}$, N. Cox ${ }^{2}$, J. Cami ${ }^{3}$, B.H. Foing ${ }^{4}$, L. Kaper ${ }^{2}$, L.

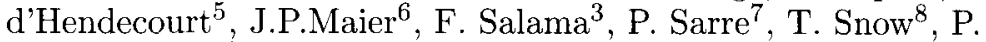 \\ Sonnentrucker ${ }^{9}$
}

${ }^{1}$ Leiden Observatory, P O Box 9513, 2300 RA Leiden, Netherlands

${ }^{2}$ Astronomical Institute "Anton Pannekoek", University of Amsterdam, Kruislaan 403, NL-1098 SJ Amsterdam, Netherlands

${ }^{3}$ Space Science Division, NASA Ames Research Center, MS 245-3, Moffett Field, CA 94035, USA

${ }^{4}$ Research Support Division, ESA, ESTEC SCI-SR, 2200 AG Noordwijk, Netherlands

${ }^{5}$ Institut d'Astrophysique Spatiale, Campus d'Orsay, 91405 Orsay, France

${ }^{6}$ Institute for Physical Chemistry, Klingelbergstrasse 80, University of Basel CH-4053, Switzerland

${ }^{7}$ School of Chemistry, University of Nottingham, University Park, Nottingham NG7 2RD, UK

${ }^{8}$ Center for Astrophysics and Space Astronomy, University of Colorado at Boulder, 389 UCB, Boulder, CO 80309-0389, USA

${ }^{9}$ Department of Physics and Astronomy, Johns Hopkins University, Charles and 34th Street, Bloomberg Center, Baltimore, MD 21218-2686, USA

\begin{abstract}
With the Ultraviolet Visual Echelle Spectrograph mounted at the Very Large Telescope, we have observed at unprecedented spectral resolution the absorption spectrum toward reddened stars in the Magellanic Clouds over the wavelength range of $3500-10500 \AA$. This range covers the strong transitions associated with neutral and charged large carbon molecules of varying sizes and structures. We report the first detection of diffuse interstellar bands (DIBs) at 5780 and $5797 \AA$ in the Small Magellanic Cloud and the variation of those DIBs toward several targets in the Large Magellanic Cloud. The variation of DIBs in the Magellanic Clouds compared with Galactic targets may be governed by a combination of the different chemical processes prevailing in low-metallicity regions and the local environmental conditions. The analysis of high-resolution absorption spectra allows us to reveal the global effects in the chemistry and recycling of cosmic dust in the Magellanic Clouds which are relevant for the chemical pathways forming large organic molecules in external galaxies.
\end{abstract}

\section{Diffuse interstellar bands in the Magellanic Clouds}

The diffuse interstellar bands (DIBs) are a large number of absorption lines between 4000-10000 $\AA$ that are superimposed on the interstellar extinction curve (Herbig 1995). In the last 75 years DIBs have been observed toward more than a 
hundred stars. The number of known DIBs steadily increases due to the higher sensitivity of detectors and is currently estimated to be several hundreds. At present, no definitive identification of any of the carriers of the DIBs exists, though large gaseous carbon molecules are strongly favored (Ehrenfreund \& Charnley 2000). There are only a handful of DIBs which are strong enough to be detected in extra-galactic systems. But DIBs have been observed toward other galaxies and in particular in several star-burst galaxies (Heckman \& Lehnert $2000)$.

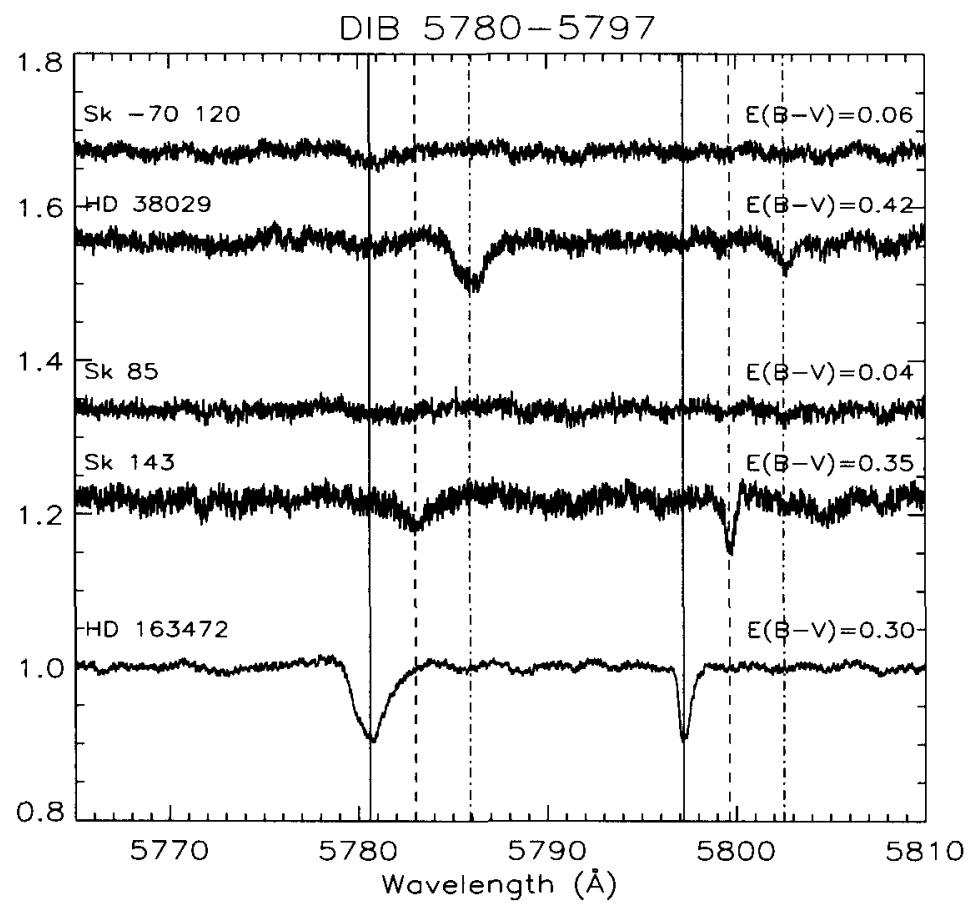

Figure 1. This figure shows spectra of the two best known DIBs, the $\lambda \lambda 5780$ and 5797 DIB. All spectra are normalized to the continuum, and shifted for display. The spectrum at the bottom shows a galactic translucent cloud source (HD 163472). The two middle spectra show an SMC target Sk 143 (AzV456) and an unreddened SMC standard Sk 85 (AzV242); the two top spectra show an LMC target (HD 38029) and an unreddened LMC standard Sk -70 120. The full vertical lines indicate the galactic rest wavelengths for those DIBs, the dashed lines indicate the wavelengths expected for these DIBs at SMC velocities, as determined from the $\mathrm{NaD}$ lines and the dash-dotted lines are the same for the LMC velocities. Both DIBs are clearly detected in both the SMC and LMC reddened targets (Ehrenfreund et al. 2002).

We reported recently the first detection of diffuse interstellar bands (DIBs) at 5780 and $5797 \AA$ in the Small Magellanic Cloud (SMC), and measured the variation of DIBs in the SMC, the Large Magellanic Cloud (LMC) and our galaxy (Ehrenfreund et al. 2002). For these observations we used the UVES spectrograph on the VLT. The interest of observing the Magellanic Clouds are the difference in metallicity (LMC: 2.5 times less and SMC: 10 times less) and the 
smaller gas-to dust ratio ( 4 and 30 times less for LMC and SMC, respectively). Furthermore these irregular galaxies are nearest neighbors ( 60 and $50 \mathrm{kpc}$ away) and certain regions within the SMC and LMC have been rather well characterized in terms of UV radiation field, extinction curve, star formation conditions and other environmental factors. In the SMC, DIBs have been exclusively observed toward Sk143, the only line-of-sight which shows a reasonable bump at $2200 \AA$ in the interstellar extinction curve (Gordon et al. 2003). This target is located in the SMC wing. Stars in the SMC bar are devoid of the $2200 \AA$ bump and show instead a high far UV linear rise in the UV part of the interstellar extinction curve. Those targets do not show evident DIBs. The LMC lines-of sight show a varying pattern of DIBs. Several stars within 30 Dor region show strong DIBs, but some stars very close by do not (Ehrenfreund et al. 2002). A combination of different chemical processes prevailing in low metallicity regions and the local environmental conditions may govern the presence, strength and absence of DIBs in these extra-galactic targets.

\section{Conclusion}

In the Magellanic Clouds environmental effects are different from those in the Milky Way. Large variations in DIB properties exist in different LMC lines-of sight. The band strengths of DIBs in the SMC (Sk143) and 30 Dor regions (LMC) show a strong linear relationship with those in the Milkyway. Studying the environmental conditions that play a role in the DIB formation may help us to constrain the identity of the carriers. The positive correlation between the presence of the UV-bump and DIBs may indicate the carbonaceous nature of the carrier. The carrier identification will give us a powerful diagnostic tool to probe and explore the diffuse ISM in our and external galaxies.

\section{References}

Ehrenfreund, P. \& Charnley, S. 2000, ARAA, 38, 427

Ehrenfreund, P. et al. 2002, ApJ, 576, L117

Gordon, K. et al. 2003, ApJ, 594, 279

Heckmann, T.M. \& Lehnert, M.D. 2000, ApJ, 537, 690

Herbig, G. 1995, ARAA, 33, 19 